\title{
Secondary Omental Torsion as a Rare Cause of Acute Abdomen in a Child and the Advantages of Laparoscopic Approach
}

\author{
Tommaso Gargano $^{1}$ Michela Maffi ${ }^{1}$ Noemi Cantone ${ }^{1}$ Francesca Destro $^{1}$ Mario Lima ${ }^{1}$ \\ ${ }^{1}$ Department of Pediatric Surgery, University of Bologna, Bologna, \\ Italy \\ Address for correspondence Tommaso Gargano, MD, Department of \\ Pediatric Surgery, University of Bologna, S. Orsola - Malpighi Hospital, \\ Massarenti 11 Street, Bologna 40138, Italy \\ Eur J Pediatr Surg Rep 2013;1:35-37. \\ (e-mail: tommaso.gargano2@unibo.it).
}

\begin{abstract}
Omental torsion is an underdiagnosed cause of acute abdomen in children. Most cases occur in adults, with only $15 \%$ presenting in children. It is estimated that omental infarct is found in approximately 0.1 to $0.5 \%$ of children undergoing operations for appendicitis. It may present with various signs and symptoms, although the clinical presentation usually mimics that of acute appendicitis. Definitive diagnosis is often established during surgery. We report a case of a 9-year-old boy who came to our attention for acute

Keywords

- omental torsion

- acute abdomen

- laparoscopy abdomen. We performed a laparoscopy-assisted procedure suspecting acute appendicitis. We therefore identified a secondary omental torsion associated with patency of the right inguinal duct and herniated omentum. Laparoscopy allowed a correct diagnosis and therapy.
\end{abstract}

\section{Introduction}

Omental torsion is a rare cause of acute abdomen. It is due to a twist of the greater omentum around its axis leading to perfusion defects and vascular impairment and later to edema, ischemia, and gangrene. ${ }^{1,2}$ Omental torsion can be either primary (idiopathic) or secondary., ${ }^{3,4}$ This condition was first described in $1851 .^{5}$ Since then only a few cases have been reported in children, ${ }^{1-10}$ and even fewer reported cases were associated with inguinal hernia. Omental torsion is responsible for $0.1 \%$ of surgical procedures performed for acute appendicitis in children. ${ }^{6}$ We report the case of a male patient with secondary omental torsion diagnosed and treated through a laparoscopy-assisted procedure.

\section{Case Report}

A 9-year-old boy came to our attention for a 24-hour history of lower and constant abdominal pain. He also complained for fever and anorexia. His medical history was uneventful with

no signs of inguinal swelling. Physical examination revealed tenderness over the lower abdominal quadrants. Biochemical analysis showed mild leukocytosis (10.730 leukocytes $/ \mathrm{mm}^{3}$ ) and augmented phlogosis markers. All other laboratory tests were normal. The patient underwent a laparoscopy-assisted procedure with a suspected diagnosis of appendicitis. We used a 10-mm umbilical operative optic and two additional 5$\mathrm{mm}$ operative trocars. Pneumoperitoneum was created with $10 \mathrm{~mm} \mathrm{Hg}$ of pressure and $1 \mathrm{~L} / \mathrm{min}$ of flow. During laparoscopic exploration, we observed the right part of the omentum fixed to the internal inguinal ring and twisted several times around its axis. The involved omentum was first reduced and then removed with the LigaSure (Covidien Italia S.p.A., Segrate - MI, Italy) (-Fig. 1). After this maneuver, a minimal patency of the inguinal duct was identified. A laparoscopyassisted incidental appendectomy was also performed. The appendix was grossly normal. We decided to avoid other procedures on the duct because of the extended phlogosis. The recovery was uneventful and the patient was discharged

(c) 2013 Georg Thieme Verlag KG Stuttgart · New York
DOI http://dx.doi.org/ $10.1055 / \mathrm{s}-0033-1345280$. ISSN 2194-7619.
March 17, 2012

accepted after revision

April 2, 2013

published online

May 9, 2013 
3 days later. The pathologist confirmed the diagnosis of omental torsion showing ischemia and necrosis. The boy is doing well 3 months after surgery ( - Fig. 1 a-d).
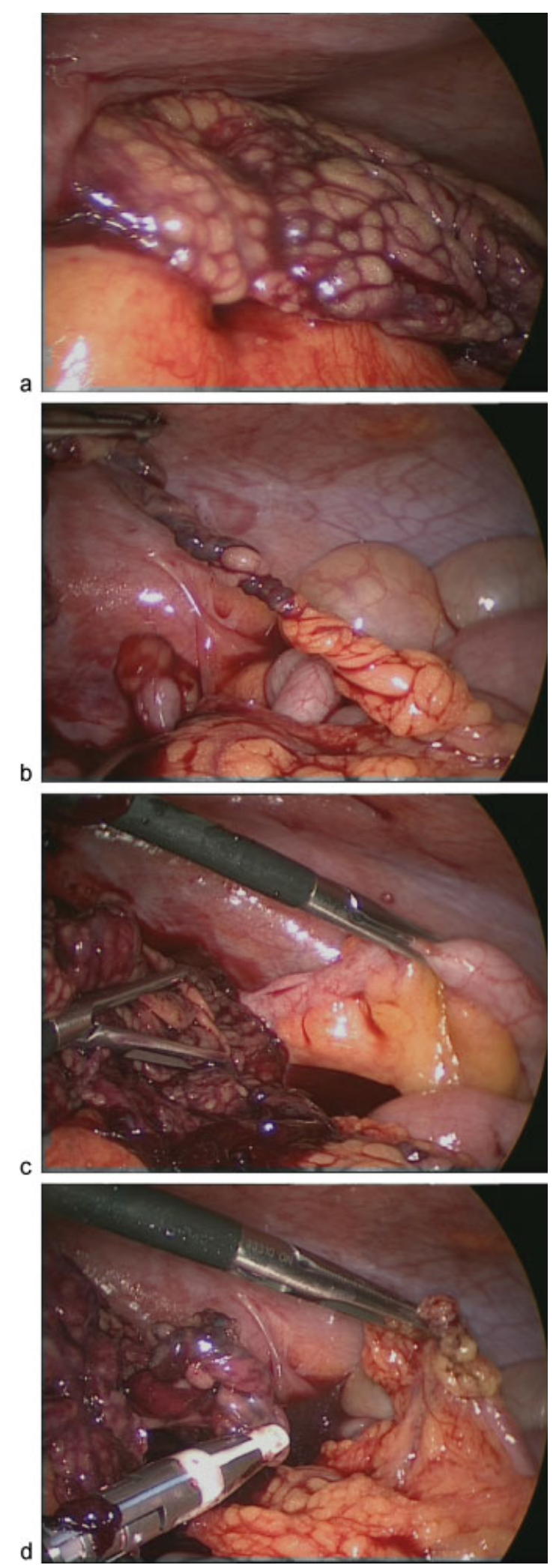

Fig. 1 Laparoscopic findings: (a) necrotic omentum fixed to internal inguinal ring; (b) omentum twisted around its axis; (c) normal appendix; and (d) omentum removal with LigaSure.

\section{Discussion}

Omental torsion is a rare condition whose preoperative diagnose may be challenging. Indeed, it does not present a specific clinical pattern. It should always be considered in the case of acute abdominal pain. The differential diagnosis includes acute appendicitis, complicated Meckel diverticulum, acute cholecystitis, and other diseases. ${ }^{3}$ Idiopathic omental torsion still lacks a certain etiology, and it is preoperatively diagnosed in only 0.6 to $4.8 \%$ of all cases. ${ }^{3,7}$ Infarction of the right side of the omentum is more frequent. This may be related to the greater length and mobility ${ }^{7}$ or to the different embryological origin with congenitally anomalous and tortuous blood supply. ${ }^{4,5}$ Bifid omentum (accessory omentum originating from a narrow route and excessive adipose tissue accumulation) and obesity (especially when body mass index is above the 95th percentile ${ }^{3}$ ) have been identified as predisposing factors. Trauma, coughing, a sudden change of body position, hyperperistalsis, or compression between the liver and the abdominal wall have been proposed as precipitating factors. ${ }^{2,8,9}$ Secondary omental torsion may be associated with abdominal affections such as cysts, tumors, inflammation, previous abdominal surgery or hernias, and other conditions that increase abdominal pressure. ${ }^{2,4,6}$ Clinical patterns are nonspecific and may include both signs and symptoms of acute abdomen (abdominal pain, abdominal tenderness, nausea and vomiting, fever, and leukocytosis) and a palpable mass (in case of large omentum involvement). Instrumental evaluation should include an abdominal ultrasonography (US). US is important to exclude other pathologies such as acute cholecystitis or pancreatitis along with laboratory tests. The twisted omentum may appear as a circular hyperechoic mass with poor vascularization. ${ }^{3-5}$ In the reported case, we decided to perform a laparoscopic exploration right away and without US evaluation because clinical evidence made us believe the acute abdomen was related to acute appendicitis. In hindsight, abdominal US could have been a good modality for initial evaluation of other potential causes of acute abdominal pain. Computed tomography (CT) is advocated by many authors as an important preoperative tool. ${ }^{3,8,11}$ We argue that CT evaluation does not affect the final management, ${ }^{2}$ and it increases $\mathrm{X}$-ray exposure to children. Otherwise, laparoscopy permits to differentiate omental torsion from other causes of acute abdomen, and it carries with it the advantages of a miniinvasive procedure. It is also a therapeutic approach. In our case, no predisposing factors could be identified and acute appendicitis was the initial clinical possibility. When the diagnosis was clear, we decided to perform an omental partial resection because conservative treatment is uncertain and it requires a huge amount of drug administration in children., ${ }^{1,8}$ The torsion was secondary to omental incarceration into the patent inguinal duct. We decided to avoid the duct closure considering that the defect was really narrow and the inflammation was extended. We therefore do not exclude the possibility to have a spontaneous closure, thanks to healing adhesions. We planned a clinical and radiological (US) followup to monitor the possible onset of hydrocele or inguinal 
hernia. We simultaneously performed appendectomy to avoid its future involvement or future diagnostic dilemma, considering the possible recurrence of omental torsion.

\section{Conclusion}

Omental torsion mimics other causes of acute abdomen, and it should be considered in the differential diagnosis. Laparoscopy provides the diagnosis and treatment with minimal morbidity and rapid recovery. The procedure also has the following advantages: abdominal cavity exploration, alternative maneuvers when required, and excellent esthetical results.

Conflict of Interest

None

\section{References}

1 Lugo B, Emil S. Omental torsion. J Pediatr Surg 2006;41(2): 458-459

2 Panagidis A, El Salam S, Al Marzauqi A, Hobeldin M, Mahomed A. The emerging role of laparoscopy in diagnosis and treatment of primary omental torsion in children. J Laparoendosc Adv Surg Tech A 2008;18(2):324-326
3 Efthimiou M, Kouritas VK, Fafoulakis F, Fotakakis K, Chatzitheofilou K. Primary omental torsion: report of two cases. Surg Today 2009;39(1):64-67

4 Valioulis I, Tzallas D, Kallintzis N. Primary torsion of the greater omentum in children - a neglected cause of acute abdomen? Eur J Pediatr Surg 2003;13(5):341-343

5 Houben $\mathrm{CH}$, Powis M, Wright VM. Segmental infarction of the omentum: a difficult diagnosis. Eur J Pediatr Surg 2003;13(1): 57-59

6 Mavridis G, Livaditi E, Baltogiannis N, Vasiliadou E, ChristopoulosGeroulanos G. Primary omental torsion in children: ten-year experience. Pediatr Surg Int 2007;23(9):879-882

7 Costi R, Cecchini S, Randone B, Violi V, Roncoroni L, Sarli L. Laparoscopic diagnosis and treatment of primary torsion of the greater omentum. Surg Laparosc Endosc Percutan Tech 2008; 18(1):102-105

8 Nubi A, McBride W, Stringel G. Primary omental infarct: conservative vs operative management in the era of ultrasound, computerized tomography, and laparoscopy. J Pediatr Surg 2009;44(5): 953-956

9 Chan KW, Chow CS, Tam YH, Lee KH. Laparoscopy: an excellent tool in the management of primary omental torsion in children. J Laparoendosc Adv Surg Tech A 2007;17(6): 821-824

10 Floyd MS Jr, Kelly BJ, Udani P, O’Donnell N, Morrison P. Abdominal pain in a 9-year-old: omental torsion masquerading as appendicitis. Br J Hosp Med (Lond) 2008;69(1):47

11 Kayan M, Sabuncuoglu MZ, Çetin M, et al. Omental torsion with left-sided inguinal hernia: a rare preoperative diagnosis. Clin Imaging 2013;37(1):173-175 